\title{
Influenza and respiratory syncytial virus infections in British Hajj pilgrims
}

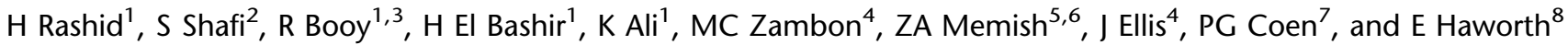 \\ ${ }^{1}$ Academic Unit of Child Health, Queen Mary University of London, London, UK; ${ }^{2}$ HPA Collaborating Laboratory, Department of Microbiology, \\ Northwick Park Hospital, Middlesex, UK; ${ }^{3}$ National Centre for Immunisation Research and Surveillance of Vaccine Preventable Disease, The \\ Children's Hospital at Westmead, The University of Sydney, Sydney, New South Wales, Australia; ${ }^{4}$ Respiratory Virus Unit, Centre for Infections, \\ Health Protection Agency, London, UK; ${ }^{5}$ Department of Medicine, King Abdulaziz Medical City, National Guard Health Affairs, Riyadh, \\ Saudi Arabia; ${ }^{6}$ Department of Infection Prevention and Control, King Abdulaziz Medical City, National Guard Health Affairs, Riyadh; \\ Saudi Arabia; ${ }^{7}$ Infection Control Office, Windeyer Institute of Medical Sciences, University College. London Hospitals NHS Trust, London, UK; \\ and ${ }^{8}$ Thames Valley Health Protection Unit, Health Protection Agency South East, Oxford, UK.
}

\section{Correspondence}

Dr H Rashid, Academic Unit of Child Health, Queen Mary University of London, Abernethy Building, 2 Newark Street, London E1 2AT, UK

E-mail: h.rashid@qmul.ac.uk

Received 10 December 2006 Revised 3 September 2007 Accepted 27 September 2007
Viral respiratory infections including influenza and respiratory syncytial virus (RSV) have been reported during the Hajj among international pilgrims. To help establish the burden of these infections at the Hajj, we set up a study to confirm these diagnoses in symptomatic British pilgrims who attended the 2005 Hajj. UK pilgrims with symptoms of upper respiratory tract infection (URTI) were invited to participate; after taking medical history, nasal swabs were collected for point-of-care testing (POCT) of influenza and for subsequent PCR analysis for influenza and RSV. Of the 205 patients recruited, 37 (18\%) were positive for either influenza or RSV. Influenza A (H3) accounted for 54\% (20/37) of the virus-positive samples, followed by RSV 24\% (9/37), influenza B 19\% (7/37), and influenza A (H1) 3\% (1/37). Of the influenza-positive cases, $29 \%(8 / 28)$ had recently had a flu immunisation. Influenza was more common in those who gave a history of contact with a pilgrim with a respiratory illness than those who did not (17 versus $9 \%)$. The overall rate of RSV was $4 \%(9 / 202)$. This study confirms that influenza and RSV cause acute respiratory infections in British Hajj pilgrims. Continuing surveillance and a programme of interventions to contain the spread of infection are needed at the Hajj, particularly when the world is preparing for an influenza pandemic.

\section{Introduction}

The Hajj is an annual pilgrimage of Muslims in Mecca, the holiest place of Islam. Every able Muslim is obliged to perform Hajj at least once in a lifetime, becoming a Hajji. Annually, more than 2 million Muslims from over 140 countries embark on the Hajj. In the year 2005, about 25,000 Hajjis travelled from the UK alone. ${ }^{1}$

Several weeks before the Hajj, pilgrims from different countries start arriving at various times, in Mecca in groups usually led by accredited tour operators. At first, they walk around the Kabah in the Grand Mosque, circling seven times (Figure 1). Later, some of them go to Medina, a city north of Mecca, where the shrine of Prophet Mohammed is situated. On the eighth day of the 'Hajj Month' they all move to Mina in a body and stay there for the next five days in tents reserved for each tour group by specified country or continent of origin. Each large tent is shared by 50-100 people. More than 2 million pilgrims move to Arafat for a day-long stay but come back to Mina after making an overnight stop in Muzdalifa. Mina, Muzdalifa, and Arafat are normally uninhabited valleys of 246, 682, and 1361 hectares, respectively, situated a few miles east of Mecca. Communicable diseases resulting from this intense crowding are well reported. $^{2}$

Respiratory illnesses are responsible for most hospital admissions at the Hajj with pneumonia being the leading cause. ${ }^{3}$ Rapidly transmissible viral respiratory infections, for example, influenza, have been reported among the pilgrims. ${ }^{4-6}$ Previous estimates suggest that $6 \%$ of pilgrims with URTI have influenza, thus, about 24,000 pilgrims could suffer from influenza infections during the Hajj each year. ${ }^{5}$

Respiratory syncytial virus, a frequent respiratory viral pathogen in elderly individuals ${ }^{7}$ shown to be responsible for $1-3 \%$ new respiratory illnesses in symptomatic international Hajj pilgrims, ${ }^{4,5}$ has not been reported previously in British pilgrims. Hajjis returning home are a source of infection,

This is an Open Access article distributed under the terms of the Creative Commons Attribution licence which permits unrestricted use, distribution, and open - ACcess reproduction in any medium, provided the original work is properly cited. 


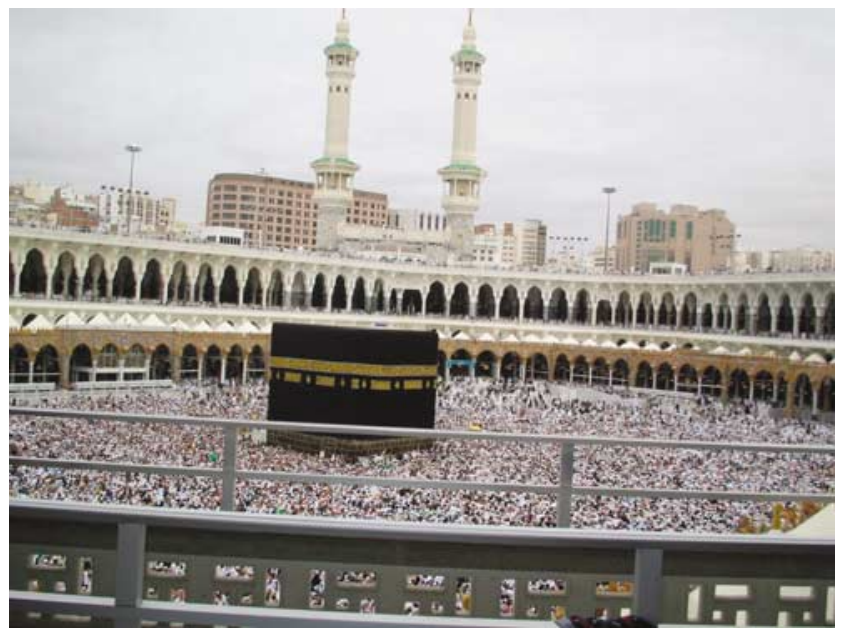

Figure 1 Pilgrims making circumambulations around the Grand Mosque in Mecca during the Hajj.

which could give rise to a global influenza outbreak as demonstrated by the outbreak of meningococcal W135 infection associated with the Hajj in the years 2000 and 2001. ${ }^{8}$ Among the European countries, Britain sends most pilgrims to the Hajj and would be expected to have the highest risk of infection transmitted by returning Hajj pilgrims. ${ }^{9}$

In this context, our aim was to establish the burden of influenza and RSV in symptomatic British pilgrims who attended the Hajj in 2005. We wished to assess the usefulness of point-of-care testing (PoCT), QuickVue ${ }^{\circledR}$ influenza test (Quidel Corporation, San Diego, CA, USA), in guiding immediate management and compared diagnosis by PoCT with subsequent analysis of respiratory samples by reverse transcription polymerase chain reaction (RT-PCR).

\section{Methods}

Ethical approval

Multicentre Research Ethics Committee (MREC) approval was obtained before commencing the study. Initial approval was for Meningococcal W135 surveillance among Hajj pilgrims, entitled, 'Meningococcal W135 carriage; enhanced surveillance among East London, South London, Mid and South Buckinghamshire UK Muslims, their household members and close contacts before and after attending the Hajj' in 2002 (Ref: MREC 02/2/12). Amendments were permitted to cover respiratory infections among Hajj pilgrims in subsequent years.

Study design

After obtaining informed written consent from each Hajji, a questionnaire was completed to include demographic details, presenting symptoms, hospital visits, history of medication, vaccination history, and risk factors such as likely contacts, shared accommodation, pre-existing diseases, previous history of Hajj, and smoking. Hajjis were coded according to their tour groups.

\section{Case definition}

UK pilgrims with symptoms of URTI such as sore throat, cough, runny nose, or fever attending the Hajj in the year 2005 .

\section{Study location}

The enhanced surveillance was undertaken with the cooperation of the British Hajj Delegation (BHD) Medical Clinic and six travel group leaders, which targeted a population of about 1000 . BHD clinic was run by British general practitioners (GPs) to provide primary medical care to the pilgrims who were staying in hotel accommodation around the vicinity of the Grand Mosque in Mecca in the build up to the Hajj.

The study was carried out in two phases: the first phase was in Mecca at the BHD clinic near the Grand Mosque on days 1 and 2 of the Hajj and the second in tents in Mina on days 3, 5,6 , and 7 . No pilgrim was recruited on day 4 , when all moved to the plain of Arafat for day-long activities as a part of the ritual.

In the first phase, pilgrims attending the BHD Medical Clinic were informed about the study and the procedure for taking samples, and were invited to participate if suffering from symptoms of URTI.

In the second phase, the team visited each group to explain the purpose of the study and the procedure for taking samples. Patients with URTI were invited to participate.

Pilgrims were examined, temperatures checked, and two nasal swabs taken.

Those with underlying diseases such as diabetes, chronic heart, lung, or kidney diseases, otherwise immunosuppressed, and those over the age of 65 years old were grouped as 'at risk'.

\section{Storage and transport of samples}

One of the swabs was used for PoCT and the other was dipped into lysis buffer (guanidinium thiocyanate and Triton X-100; Roche Diagnostics, Mannheim, Germany; Code 03246779001) for storage and subsequent transportation to the UK. Throughout the storage and transportation, a cold chain of -20 to $4{ }^{\circ} \mathrm{C}$ was maintained.

\section{Patient management}

Treatment with oseltamivir was offered to symptomatic Hajjis who were positive for influenza by PoCT within $48 \mathrm{~h}$ of onset of symptoms.

\section{Molecular diagnosis}

Molecular diagnosis of influenza and RSV was carried out at the Centre for Infections at the Health Protection Agency (London, UK) by RT-PCR as previously described. ${ }^{10-12}$ 


\section{Follow-up}

Hajjis were contacted within 12 weeks of the Hajj to document complications of their viral infections. Vaccination history was verified by primary care providers.

\section{Data analysis}

All statistical analyses were carried out in STATA 9.0. In this enhanced surveillance study, infections are presented as proportions tested in the form of percentages. Tests of significance are Fisher's exact tests.

\section{Results}

\section{Demographic details}

We recruited 205 Hajjis with symptoms of URTI, 50\% (103/ 205) of whom were seen at BHD clinic, and the remaining 102 patients were seen in the Mina tents.

Nasal swabs were collected from 205 patients and 202 samples were analysed (two patients declined to provide swabs and one sample was lost).

Hajjis came from all over the UK including Northern Ireland. Ten (5\%) non-British Hajjis staying with British Hajjis were also included in the study. Ninety per cent (185/ 205) were men. Ages ranged from $1 \frac{1}{2}$ to 83 years (mean and median: 44 years). Ethnic origin was 80\% Indian (165/205), $5 \%$ African (11/205), 2\% Arabian (4/205), 1\% British (3/205), $6 \%$ other $(13 / 205)$, and $4 \%$ unknown $(9 / 205)$ (Table 1$)$.

\section{Epidemiology and virology}

The common presenting symptoms of the Hajjis with URTI were $80 \%$ sore throat (163/205), 74\% cough (151/205), 69\% runny nose (142/205), and 53\% fever (109/205), whose

Table 1 Distribution of influenza and RSV infections among different categories of patient

\begin{tabular}{lccc}
\hline Category & $\begin{array}{l}\text { Sample } \\
\text { size }\end{array}$ & $\begin{array}{l}\text { Influenza-positive } \\
\text { cases (\%) }\end{array}$ & $\begin{array}{l}\text { RSV-positive } \\
\text { cases (\%) }\end{array}$ \\
\hline $\begin{array}{l}\text { Ethnicity } \\
\text { Indian }\end{array}$ & 165 & $19(12 \%)$ & $9(5 \%)$ \\
Arab & 4 & $0(0 \%)$ & $0(0 \%)$ \\
African & 11 & $3(27 \%)$ & $0(0 \%)$ \\
British & 3 & $0(0 \%)$ & $0(0 \%)$ \\
Others & 13 & $6(46 \%)$ & $0(0 \%)$ \\
Unknown & 6 & $0(0 \%)$ & $0(0 \%)$ \\
Age (years) & & & $0(0 \%)$ \\
$<16$ & 3 & $0(0 \%)$ & $2(2 \%)$ \\
16-44 & 101 & $8(17 \%)$ & $5(6 \%)$ \\
$45-64$ & 80 & $3(17 \%)$ & $2(11 \%)$ \\
$>64$ & 18 & & $8(4 \%)$ \\
Risk category & & $5(9 \%)$ & $1(5 \%)$ \\
At risk & 53 & $23(15 \%)$ & $4(3 \%)$ \\
Not at risk & 149 & & \\
Gender & & $23(13 \%)$ & \\
Male & 182 & $5(25 \%)$ & \\
Female & 20 & & \\
\hline
\end{tabular}

positive predictive values for influenza were $92,82,75$, and $71 \%$, respectively 202 .

Nine of 202 (4\%) patients were positive for influenza by PoCT at the time of consultation. All these positive patients were in the BHD clinic group.

Out of 202 patients, 28 (14\%) were positive for influenza by RT-PCR test, 20 for influenza A H3 (71\%), seven for influenza B (25\%), and one for influenza A H1 (4\%). PoCT did not detect the virus in $71 \%(20 / 28)$ of RT-PCR-positive cases. One PoCT gave a false-positive result (if PCR was correct), giving a sensitivity and specificity of PoCT as 29 and $99 \%$, and positive and negative predictive values as 89 and 90\%, respectively. All other PoCT-positive cases were identified as influenza A H3 or H1 on PCR testing. All of them presented four days or more after arrival at the Hajj.

Nine out of the 202 (4\%) patients were positive for RSV A by RT-PCR, and all presented eight days or more after arrival at the Hajj.

Fifty-eight per cent $(118 / 202)$ of patients gave a history of contact with other ill Hajjis, 37\% (74/202) gave no history of contact, and 5\% (10/202) were not sure about contact with an ill pilgrim. The frequency of influenza diagnosis was 17\% (20/ 118) among those who reported contact with URTI compared with $9 \%(7 / 74)$ who did not report contact with URTI $(P=0.15)$.

Sixty (30\%) of those with respiratory infection were treated with antibiotics, the commonly used being amoxicillin $(15 \%, n=31)$, out of which $21(10 \%)$ were in 'at-risk' group. Nine $(4 \%)$ patients were treated with oseltamivir, and two $(1 \%)$ were in 'at-risk' group.

\section{Vaccination status}

Of the 202 patients tested, 56 (28\%) received influenza vaccine before travelling, 143 (71\%) definitely did not, and three $(1 \%)$ were not sure if they had been vaccinated.

The proportion of influenza-positive cases was $14 \%(8 / 56$, $20 / 144)$ in both vaccinated and unvaccinated patients.

Twenty-nine per cent $(8 / 28)$ of patients with positive diagnosis of influenza had confirmed history of vaccination.

\section{'At-risk' groups \\ Twenty-six per cent (53/202) fell into the 'at-risk' category, one- third $(n=18)$ just because of being aged $>65$ years. Influenza vaccine uptake rates were $47 \%$ (25/53) in the 'at-risk' group and $21 \%(31 / 149)$ in the 'not at-risk' group $(P<0.01)$. Confirmed influenza among 'at-risk' and 'not at-risk' groups was $9 \%(5 / 53)$ and $15 \%(23 / 149)$, respectively $(P=0.28)$ (Table 1$)$. Fifty per cent $(9 / 18)$ of pilgrims $\geqslant 65$ and $25 \%(46 / 184)<65$ years of age were vaccinated. The influenza infection rates in these groups were 17 and $14 \%$, respectively $(P=0.72)$. \\ Fifteen per cent $(31 / 202)$ of Hajjis reported that they were regular smokers. Six per cent of smokers (2/31) and 15\% of non-smokers $(26 / 171)$ tested positive for influenza $(P=0.19)$.}

\section{Post-Hajj follow-up}

None of the pilgrims contacted after returning from the Hajj had complications attributable to influenza or RSV infection. 


\section{Discussion}

The burden of influenza in the sample of symptomatic British pilgrims tested at the 2005 Hajj was $14 \%$ by RT-PCR and $4 \%$ by PoCT, which compares with $6 \%$ found previously among international pilgrims by virus culture. ${ }^{5}$ Influenza $\mathrm{A}$ accounted for $75 \%(21 / 28)$ and influenza B for $25 \%(7 / 28)$ of positive flu results in this study in contrast to previous findings of Balkhy et al., ${ }^{5}$ in which 90\% (27/30) were found to have had influenza B and 10\% (3/30) influenza A. Circulating influenza strains differed in the two seasons, influenza B was predominant in 2003, while influenza A (H3) was predominant in 2005. ${ }^{13,14}$ All patients with influenza presented four days or more, and all patients with RSV presented eight days or more after reaching Mecca, suggesting that the infections were acquired at the Hajj.

In this study, the sensitivity of PoCT against RT-PCR was much lower than in other studies (29\% compared with, for example, 86 and 84\%) that had measured its sensitivity against viral culture, ${ }^{15,16}$ although it had a very high specificity (99\%) and positive predictive value. Both the superiority of RT-PCR, and type and timing of the swab tested may have influenced the sensitivity of PoCT. Studies that used nasopharyngeal aspirates or washes (instead of nose swabs) had generally higher sensitivities, and the decreasing viral load after the fourth day of symptoms may reduce PoCT sensitivity. ${ }^{16,17}$ Our samples were collected within one week of onset of symptoms; we could expect higher sensitivity of the PoCT if all swabs were collected within four days.

The UK National Institute for Health and Clinical Excellence recommends that oseltamivir be used as postexposure prophylaxis of influenza in at-risk people aged $\geqslant 13$ years, who are not effectively protected by immunisation. ${ }^{18}$ The logistics of antiviral distribution at the Hajj should be explored in future studies.

Although it seems that vaccination did not confer adequate protection against influenza (both groups had the same rate), it is not possible to draw any conclusion about vaccine efficacy from this study because the groups were not comparable; that is, a large number of those immunised were 'at-risk' individuals, whereas the majority of those not immunised were healthy adults, and vaccine failure can not be ruled out. However, it is likely that there would have been more confirmed influenza in the British Hajj pilgrims if none had been vaccinated.

Two previous studies established by virus culture that the rates of RSV among symptomatic pilgrims were 3 and 1\%, respectively, in 1991-1992 and 2003 Hajj seasons. ${ }^{4,5}$ The slightly higher rate (4\%) in our study could be due to superiority of RT-PCR to virus culture or due to seasonal variation. Although the proportion of RSV infection at the Hajj is lower than that of influenza, there is potential for returning pilgrims to introduce this infection to their households including children. ${ }^{19}$

This study shows the feasibility of enhanced surveillance at the Hajj and, if strengthened year on year, could help answer some outstanding questions on the effectiveness of influenza immunisation, the use of antivirals, and the review of flu immunisation policy, in comparison with WHO guidance and the use of influenza vaccine recommended in Saudi Arabia for Hajj pilgrims. ${ }^{2}$

\section{Conclusions}

This study confirms influenza and RSV infections among British Hajj pilgrims and suggests a higher rate than reported among international pilgrims in previous years. Continuing and better surveillance at the Hajj is needed, especially now when an influenza pandemic threatens the world. More research into the use of antivirals and protective equipment in such a mixed and crowded setting, preferably prospectively, is also necessary to improve risk assessment and the effectiveness of interventions.

\section{Acknowledgements}

We thank all the pilgrims for their participation, as well as British Hajj Delegation led by Lord Patel of Blackburn for allowing us to run clinics with their team and the staff of Foreign and Commonwealth Office for their logistic support, and European Hajj Mission for assisting us in recruiting participants.

\section{Author contributions}

RB, HEB, and EH conceived and designed the study. RB and HEB obtained ethical approval. RB oversaw the study and is the guarantor. SS co-ordinated the field study. HR, KA, SS, and ZAM collected data and samples. MCZ and JE did the laboratory analysis. PGC supervised the statistical analyses. HR, RB, SS, EH, JE, MCZ, and ZAM drafted the paper.

\section{Competing Interests}

SS chaired the Health and Medical Committee of the Muslim Council of Britain (until August 2006). RB has received support from Roche, CSL, and Sanofi to attend scientific meetings.

\section{Ethical approval}

Described in the Methods.

Funding

This work was funded by the UK Department of Health.

Provenance and peer review

Not commissioned; externally peer-reviewed.

\section{References}

1 The Saudi Arabia Information Resource. 7 January 2005; available at http://www.saudinf.com/main/y7743.htm, accessed 28 August 2007.

2 Ahmed QA, Arabi YM, Memish ZA. Health risks at the Hajj. Lancet 2006;367:1008-15. 
3 Al-Ghamdi SM, Akbar HO, Qari YA, Fathaldin OA, Al-Rashed RS. Pattern of admission to hospitals during muslim pilgrimage (Haji). Saudi Med J 2003;24:1073-6.

4 El-Sheikh SM, El-Assouli SM, Mohammed KA, Albar M. Bacteria and viruses that cause respiratory tract infections during the pilgrimage (Haj) season in Makkah, Saudi Arabia. Trop Med Int Health 1998;3:205-9.

5 Balkhy HH, Memish ZA, Bafaqeer S, Almuneef MA. Influenza a common viral infection among Haji pilgrims: time for routine surveillance and vaccination. J Travel Med 2004;11:82-6.

6 El Bashir H, Haworth E, Zambon M, Shafi S, Zuckerman J, Booy R. Influenza among UK pilgrims to Haji, 2003. Emerg Infect Dis 2004;10:1882-3.

7 Falsey AR, Walsh EE. Viral pneumonia in older adults. Clin Infect Dis 2006;42:518-24.

8 Hahne SJ, Gray SJ, Aguilera JF, Crowcroft NS, Nichols T, et al. W135 meningococcal disease in England and Wales associated with Haji 2000 and 2001. Lancet 2002;359:582-3.

9 Shafi S, Memish ZA, Gatrad AR, Sheikh A. Hajj 2006: communicable disease and other health risks and current official guidance for pilgrims. Euro Surveill 2005;10:E051215.2.

10 Espy MJ, Rys PN, Wold AD, Uhl JR, Sloan LM, Jenkins GD, et al. Detection of herpes simplex virus DNA in genital and dermal specimens by LightCycler PCR after extraction using the IsoQuick, MagNA Pure, and BioRobot 9604 methods. J Clin Microbiol 2001;39:2233-6.

11 Ellis JS, Zambon MC. Combined PCR-heteroduplex mobility assay for detection and differentiation of influenza A viruses from different animal species. J Clin Microbiol 2001;39:4097-102.
12 Stockton J, Ellis JS, Saville M, Clewley JP, Zambon MC. Multiplex PCR for typing and subtyping influenza and respiratory syncytial viruses. J Clin Microbiol 1998;36:2990-5.

13 World Health Organization. Influenza. Wkly Epidemiol Rec 2003;78:57-64. Available at http://www.who.int/wer/2003, accessed 28 August 2007.

14 World Health Organization. Influenza in the world Wkly Epidemiol Rec 2005;80:77-84. Available at URL: http:// www.who.int/wer/2005, accessed 28 August 2007.

15 Ruest A, Michaud S, Deslandes S, Frost EH. Comparison of the Directigen flu $\mathrm{A}+\mathrm{B}$ test, the QuickVue influenza test, and clinical case definition to viral culture and reverse transcription-PCR for rapid diagnosis of influenza virus infection. I Clin Microbiol 2003;41:3487-93.

16 Yamazaki M, Mitamura K, Kimura K, Komiyama O, Nirasawa M, Yamamoto $\mathrm{K}$, et al. [Clinical evaluation of an immunochromatography test for rapid diagnosis of influenza]. Kansenshogaku Zasshi 2001;75:1047-53.

17 Poehling KA, Griffin MR, Dittus RS, Tang YW, Holland K, Li H, et al. Bedside diagnosis of influenzavirus infections in hospitalized children. Pediatrics 2002;110 (1 Part 1): 83-8.

18 National Institute for Health and Clinical Excellence. Guidance on the Use of Oseltamivir and Amantadine for the Prophylaxis of Influenza. National Institute for Health and Clinical Excellence, 24 September 2003; available at URL: http://www. nice.org.uk/page.aspx $?=$ TA067guidance, accessed 28 August 2007.

19 Musher DM. How contagious are common respiratory tract infections? N Engl J Med 2003;348:1256-66. 